\title{
Une insuffisance ventriculaire droite chronique révélant un volumineux kyste hydatique compressif au service de cardiologie de l'hôpital Aristide le Dantec (Dakar, Sénégal)
}

\author{
Chronic Right Ventricular Failure Revealing a Large Compressive Hydatid Cyst at \\ the Cardiology Department of the Aristide le Dantec Hospital (Dakar, Senegal)
}

\author{
S.A. Sarr · F. Aw $\cdot$ M. Ndiaye $\cdot$ J. Mingou $\cdot$ M. Bodian $\cdot$ M. Dioum $\cdot$ A. Alassane Ngaidé $\cdot$ M. Bamba Ndiaye $\cdot$ \\ A. Kane $\cdot$ M. Diao $\cdot$ S. Abdou, Ba
}

Reçu le 22 avril 2019; accepté le 29 octobre 2019

(C) Société de pathologie exotique et Lavoisier SAS 2019

\begin{abstract}
Résumé La localisation péricardique d'Echinococcus granulosus en l'absence d'une autre atteinte cardiaque est extrêmement rare. Son évolution spontanée est grave du fait du risque de rupture du kyste hydatique. Nous rapportons le cas d'une patiente de 65 ans, reçue pour des œdèmes des membres inferieurs qui évoluaient depuis 4 mois. L'examen physique notait un souffle d'insuffisance tricuspide $3 / 6$ et un syndrome d'insuffisance cardiaque droite. L'échocardiographie transthoracique a objectivé une masse intra-péricardique dont le contenu paraissait liquidien avec des plages hyperéchogènes. Elle mesurait $86 \times 61 \mathrm{~mm}$ et comprimait de manière importante les cavités droites. La tomodensitométrie thoracique a conclu à un aspect en faveur d'un kyste hydatique (GHARBI V) intrapéricardique vieilli avec effet de masse sur les cavités droites. La sérologie hydatique était négative. La patiente n'a pas accepté la cure chirurgicale. Elle a été mise sous albendazole et traitement diurétique. L'évolution a été fatale après deux mois de suivi.
\end{abstract}

Mots clés Kyste $\cdot$ Hydatidose $\cdot$ Péricarde Compression · Insuffisance cardiaque · Hôpital · Dakar · Sénégal · Afrique intertropicale

\footnotetext{
S.A. Sarr · F. Aw $(\bowtie) \cdot$ M. Ndiaye $\cdot$ J. Mingou $\cdot$ M. Bodian

M. Bamba Ndiaye $\cdot$ A. Kane $\cdot$ M. Diao $\cdot$ S. Abdou

Service de cardiologie du Centre hospitalier universitaire Aristide

Le Dantec, Dakar, Sénégal

e-mail : fatoukineaw@yahoo.fr

M. Dioum

Service de cardiologie du Centre hospitalier national de Fann, Dakar, Sénégal
}

\author{
A. Alassane Ngaidé \\ Service de cardiologie du Centre hospitalier national de Grand \\ Yoff, Dakar, Sénégal
}

\begin{abstract}
Hydatidosis, an anthropozoonosis caused by the development of the Echinococcus granulosus larva in human, is a parasitic infection that is endemic in many countries. Pericardial localization in the absence of other cardiac involvement is extremely rare. Its spontaneous evolution is serious because of the risk of rupture. We report the case of a 65 -year-old patient, who received limb edema that had been going on for 4 months, and in whom the physical examination noted a $3 / 6$ tricuspid regurgitation and a right heart failure syndrome. Transthoracic echocardiography revealed an intra-pericardial mass whose contents appeared fluid with hyperechogenic area. It measured $86 \times 61 \mathrm{~mm}$ and significantly compressed the straight cavities. Thoracic computed tomography showed an appearance in favor of an aged intrapericardic hydatid cyst (GHARBI V) with mass effect on the right cavities. The hydatid serology was negative. The patient did not accept the surgical cure. She had been put on albendazole and diuretic treatment. Fatal outcome was reported after two-month follow-up.
\end{abstract}

Keywords Cyst · Hydatidosis · Pericardium · Compression · Heart failure $\cdot$ Hospital $\cdot$ Dakar $\cdot$ Senegal $\cdot$ Sub-Saharan Africa

\section{Introduction}

On ne connaît pas précisément les raisons de la rareté de l'hydatidose humaine en Afrique de l'Ouest et australe, alors que la maladie est présente chez le bétail [8]. L'hypothèse de l'existence d'une souche d'E. granulosus particulière a été évoquée. Sa prévalence chez l'homme n'est pas connue au Sénégal, mais quelques rares cas ont été rapportés dans la littérature $[14,18]$. La localisation péricardique sans atteinte 
cardiaque est extrêmement rare, y compris en pays d'endémie $[11,15]$. Nous rapportons un cas d'hydatidose péricardique révélée par une insuffisance ventriculaire droite chronique.

\section{Observation}

Il s'agit d'une femme de 65 ans, hypertendue de découverte récente, vivant en zone rurale (Kaolack région du Sénégal), reçue au service de cardiologie de l'hôpital Aristide le Dantec (Dakar, Sénégal) pour des œdèmes des membres inférieurs d'installation progressive qui évoluaient depuis 4 mois.

L'état général était altéré, la température était de $37,6^{\circ} \mathrm{C}$, la fréquence cardiaque de 64 cycles par minute et la pression artérielle à 140/90 mm Hg.

L'examen physique notait un assourdissement des bruits du cœur, un souffle systolique tricuspidien d'intensité $3 / 6$, une turgescence spontanée des veines jugulaires, une hépatomégalie douloureuse à surface antérieure lisse avec flèche à $16 \mathrm{~cm}$ et des œdèmes des membres inférieurs, bilatéraux, mous, indolores, prenant le godet et remontant aux genoux.

Sur le plan biologique, elle avait une anémie normochrome normocytaire à $10,8 \mathrm{~g} / \mathrm{dl}$ : il n'y avait pas d'hyperéosinophilie. La fonction rénale et le bilan hépatique étaient normaux. La sérologie hydatique était négative.

L'ECG inscrivait un rythme sinusal régulier à 65 cycles par minute, un axe du QRS à $+130^{\circ}$ un bloc de branche droit incomplet et des ondes négatives en antéro-septo-apical.
L'échocardiographie transthoracique objectivait une masse intrapéricardique dont le contenu paraissait liquidien avec quelques plages hyperéchogènes compatibles avec des calcifications (Fig. 1). Elle mesurait $86 \times 61 \mathrm{~mm}$ et comprimait de manière importante l'oreillette droite et surtout le ventricule droit, entrainant un gradient intraventriculaire droit dont la valeur moyenne était de $7 \mathrm{~mm} \mathrm{Hg}$. Cette structure possédait une enveloppe épaisse, calcifiée par endroit.

La tomodensitométrie thoracique a conclu à un aspect en faveur d'un kyste hydatique (GHARBI V) intrapéricardique vieilli avec un effet de masse sur les cavités droites (Fig. 2).

Une chirurgie a été proposée, mais la patiente n'était pas consentante. Elle a été mise sous albendazole $(15 \mathrm{mg} / \mathrm{kg})$ en plus d'un traitement diurétique. L'évolution fut fatale après deux mois de suivi.

\section{Discussion}

L'hydatidose cardio-péricardique est rare y compris dans les pays d'endémie. Elle constitue en effet 0,5 à $2 \%$ de l'ensemble des localisations hydatiques [6] et 2,6\% de celles intrathoraciques [16]. Sa gravité est liée aux complications représentées essentiellement par la rupture [11]. Cette rupture est à l'origine d'une dissémination systémique ou pulmonaire lorsqu'elle se fait dans les cavités cardiaques. S'il s'agit d'une rupture dans le péricarde, il en résultera une tamponnade.

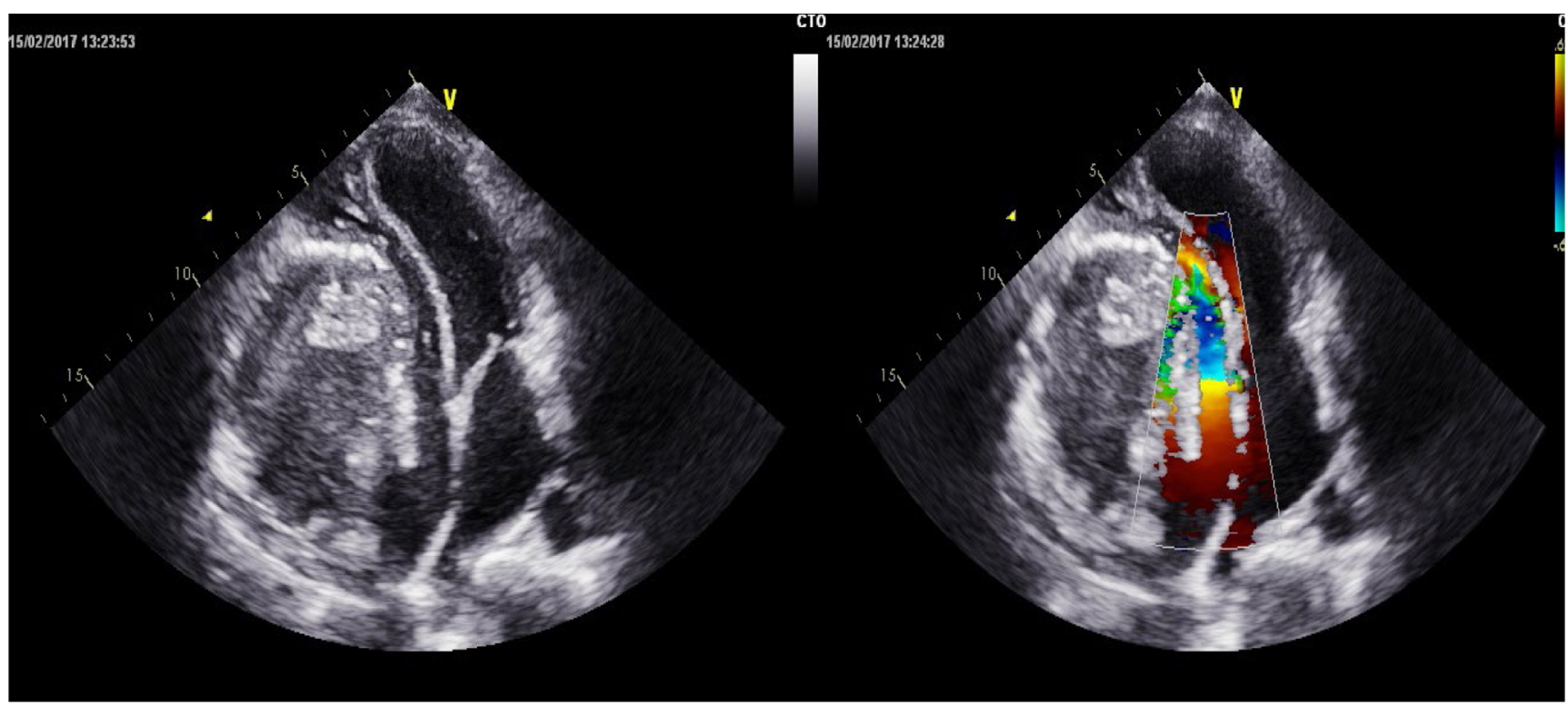

Fig. 1 Images d'échocardiographie Doppler montrant la masse péricardique, les calcifications au niveau de l'enveloppe et en son sein (gauche) ; au mode Doppler couleur, accélération du flux de remplissage en rapport avec la compression extrinsèque (à droite) / Doppler echocardiography images indicating the pericardial mass, and calcifications at the envelope and inside (left); in color Doppler mode, acceleration of the filling flow with extrinsic compression (right) 

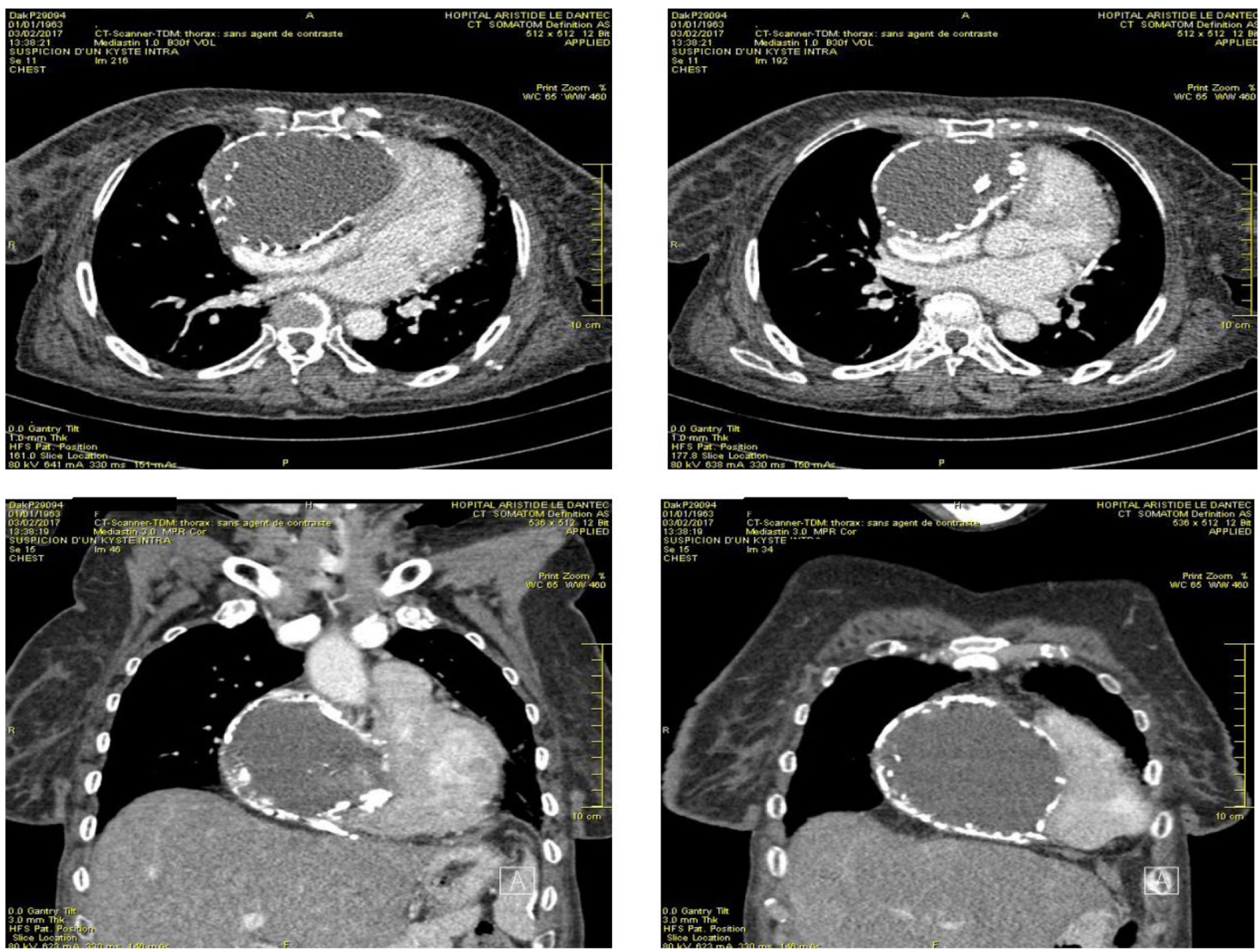

Fig. 2 Images de tomodensitométrie thoracique réalisée en mode volumique de coupes inframillimétriques avec injection : formation ovalaire intra péricardique de $102 \mathrm{~mm}$ sur son plus grand axe et $33 \mathrm{UH}$ de densité spontanée ; présence de nodules de densité tissulaire, internes et pariétales calcifiées ; paroi épaisse et en majorité calcifiée. Absence de prise de contraste interne / Thoracic computed tomography images performed in sub-millimeter volume mode with injection: intra-pericardial oval formation of $102 \mathrm{~mm}$ on its largest axis and 33 UH of spontaneous density; presence of nodules of calcified tissue, internal and parietal density; thick wall and mostly calcified. Absence of internal contrast enhancement

Le KH du cœur est généralement primitif et unique [7] comme noté dans notre cas. Il se localise préférentiellement au niveau du VG, du septum interventriculaire, du ventricule droit, des oreillettes et enfin du péricarde [11,15]. Le kyste péricardique est secondaire le plus souvent à la rupture d'un kyste hydatique du VG [11]. A ce jour nous n'avons pas retrouvé de cas de $\mathrm{KH}$ de siège cardiaque rapporté dans la littérature sénégalaise, mais des cas de KH pleural et hépatique ont été publiés $[4,14,18]$

Sur le plan clinique le KH du cœur est le plus souvent de découverte fortuite, car asymptomatique le plus souvent à la radiographie thoracique ou à l'échocardiographie [13]. Dans notre cas, il s'agissait d'un tableau d'insuffisance cardiaque droite liée à une compression extrinsèque. Cet effet s'explique aisément par le volume important du kyste et l'inex- tensibilité du péricarde. La compression était si importante qu'il y avait un gradient de pression intra-ventriculaire droit.

Sur le plan biologique, l'hyperéosinophilie sanguine est observée dans 20 à $30 \%$ des cas en phase d'invasion et au cours des ruptures de kystes [6]. Le diagnostic indirect repose sur les réactions sérologiques par les méthodes immunologiques. Si elles sont positives, avec un taux significatif, le diagnostic est retenu. Par contre, leur négativité ne permet pas d'exclure le diagnostic [2].

Les arguments diagnostiques sont aussi fournis par l'imagerie. L'échocardiographie transthoracique est l'examen de première intention dans le diagnostic de l'hydatidose cardiaque [6]. Elle montre une formation anéchogène à paroi fine avec décollement de membrane ou aspect multivésiculaire qui sont hautement évocateurs de l'origine hydatique. 
Elle permet de préciser la localisation, les rapports de la lésion kystique, la présence d'un épanchement péricardique ou pleural associé. Chez notre patiente, elle donnait déjà des arguments de chronicité avec les plages de calcification intrakystiques.

La tomodensitométrie volumique multicoupe a un intérêt multiple notamment pour un bilan morphologique locorégional très précis $[3,4]$, une étude des rapports du kyste avec les cavités cardiaques et avec les vaisseaux coronaires. Le kyste hydatique se présente comme une formation arrondie, hypodense homogène, uniloculaire à paroi fine le plus souvent, non rehaussée par le produit de contraste. La mise en évidence de calcifications pariétales comme dans notre cas est en faveur du diagnostic d'hydatidose [17], malgré l'absence de confirmation anatomopathologique et/ou parasitologique. Elle montre parfois un aspect multivésiculaire du kyste qui est un élément presque pathognomonique de l'hydatidose [5,11].

Le traitement des kystes hydatiques du péricarde est chirurgical. Il consiste en une excision des kystes pour éviter les complications [9]. Le traitement médical représente un traitement substitutif pour les patients non opérables du fait de kystes hydatiques trop nombreux, ou de terrain débilité. Il peut être complémentaire à la chirurgie lorsqu'il y a risque de dissémination. Le produit le plus utilisé est l'albendazole à la dose de 10 à $15 \mathrm{mg} / \mathrm{kg}$ par jour par cures d'un mois espacées de 15 jours pendant six mois [9].

\section{Conclusion}

L'hydatidose cardio-péricardique est une affection très rare surtout dans nos régions. En règle d'évolution latente, elle peut être à l'origine de symptômes du fait de ses complications. À côté de la compression aiguë définissant la tamponnade peut exister une complication moins connue qui est l'insuffisance cardiaque droite par compression chronique des cavités droites.

Conflits d'intérêts Les auteurs déclarent ne pas avoir de liens d'intérêts.

\section{Références}

1. Ambrosi P, Mesana T, Habib G, et al (1992) Extension intraauriculaire droite d'un kyste hydatique simulant une thrombose cardiaque. Arch Mal Cœur 85:909-12

2. Aubry P (2013) Hydatidose ou kyste hydatique; Actualités 2013. Méd Trop 4-5

3. Baqué J, Huart V, Pierrot JM, et al (2003) Kyste hydatique du septum interventri- culaire du cœur : aspect en scanner multibarettes et en IRM. J Radiol 84(5):614-6

4. Badji NF, NDong B, AkpoG, et al (2017) Apport de l'imagerie dans le diagnostic de kyste hydatique pleural primitif: à propos de deux cas. Mali Médical, 31(4):33-6

5. Ben-Hamda K, Maatouk F, Ben-Farhat M, et al (2003) Eighteenyear experience with echinococcosus of the heart: clinical and echocardiographic features in 14 patients. Int J Cardiol 91(2-3):145-51.

6. Ben Khalfallah A (1996) Apport de l'échocardiographie transœsophagienne dans le diagnostic du kyste hydatique du cœur : à propos d'un cas. Maghreb Méd 1996:305

7. Challaoui M, Bouhouch R, Akjouj M, et al (2003) Hydatidose péricardique : à propos de 3 observations. J Radiol 84(3):329-31

8. Carmoi T, Farthouat P, Nicolas X, et al (2008) Kystes hydatiques du foie. Hépathologie doi : 10.1016/S1155-1976(08)46517-8

9. El Karimi S, Ouldelgadia N, Gacem H, et al (2014) Tamponnade révélant un kyste hydatique intra-péricardique un cas. Ann Cardiol Angeiol doi : 10.1016/j.ancard.2012.01.003

10. El Kouby A, Vaillant A, Comet B, et al (1990) L'hydatidose cardiaque. Revue de la littérature récente. À propos de 15 cas. Ann Chir Thorac Cardiovasc 44(8):603-10

11. El Majhad A, Lachhab A, Charradi R, et al (2011) Apport de l'imagerie par résonance magnétique (IRM) dans le diagnostic du kyste hydatique cardiaque. East Mediterr Heart J 17(12): 996-1000. doi: 10.26719/2011.17.12.996

12. Ghrairi H, Khouadja MA, Abouda M, et al (2005) Kyste hydatique du cœur et des vaisseaux, 4 observations. Presse Méd 34 (2 Pt 1):101-4

13. Hagui A, Saidi I, Dahmani R, et al (2012) Kyste hydatique intramyocardique de découverte fortuite. Tunisie Méd 90(11):829-30

14. batchou Ngahane BH, Toure NO, Diarra O, Diatta A (2004) Pneumothorax compressif compliquant Une hydatidose pleurale. Rev Pneumol Trop 1:5-6

15. Peker RO, Sabuncu T, Kumbasar U, et al (2015) A giant hydatid cyst atypically located in the left ventricle. Turk J Pediatr 57 (3):308-10.

16. Perez-Gomez F, Duran H, Tamames S, et al (1973) Cardiac echinococcosis: clinical picture and complications. Br Heart J 35 (12):1326-31. doi: 10.1136/hrt.35.12.1326

17. Oueslati S, Saïd W, Saaidi I, et al (2006) Imagerie du kyste hydatique du cœur. À propos de 8 observations. Presse Méd 35(78):1162-6. doi : PM08-2006-35-7-8-0755-4982-101019-200604916

18. Touré AO, KâI, Sarr IS et al (2013) Kyste hydatique de la rate: à propos d'un cas. Pan Afr Med J. 14: 92. doi : 10.11604/ pamj.2013.14.92.2343 